Original Paper

\title{
Long Noncoding RNA SNHG16 Promotes Cell Proliferation by Sponging MicroRNA-205 and Upregulating ZEB1 Expression in Osteosarcoma
}

\author{
Chenlei Zhu ${ }^{\mathrm{a}} \quad$ Dong Cheng $^{\mathrm{a}} \quad$ Xubin Qiua $\quad$ Ming Zhuanga ${ }^{\mathrm{a}} \quad$ Zhiwei Liu \\ aDepartment of Orthopedics, The Third Affiliated Hospital of Soochow University, Changzhou, China
}

\section{Key Words}

$\mathrm{SNHG16} \cdot \mathrm{miR}-205 \cdot \mathrm{ZEB} 1 \cdot$ Osteosarcoma

\begin{abstract}
Background/Aims: Long noncoding RNAs (IncRNAs) have been a research hotspot, as they play important roles in tumor development. However, their expression pattern and biological function in osteosarcoma have not yet been clarified. Methods: Differentially expressed IncRNAs in osteosarcoma and paracarcinoma tissues were identified by screening an IncRNA microarray, and candidate IncRNAs were verified by quantitative real-time PCR (qRT-PCR). A series of bioinformatics and molecular biological methods were adopted to investigate the interaction among IncRNA, microRNA (miRNA), and miRNA target genes during the development and occurrence of osteosarcoma. Cell viability was measured using a Cell Counting Kit- 8 assay. Results: Chip microarray screening combined with the validation of differentially expressed candidate IncRNAs showed that the IncRNA small nucleolar RNA host gene 16 (SNHG16) had the largest fold change. SNHG16 was highly expressed in osteosarcoma tissues and cell lines, and its downregulation led to the suppressed proliferation of osteosarcoma cells. Further investigations revealed that SNHG16 could upregulate zinc finger E-box-binding homeobox 1 (ZEB1) expression by acting as an endogenous sponge of miR-205. Moreover, rescue assays proved that the effects of SNHG16 on the proliferation of osteosarcoma cells were dependent on miR-205. Conclusion: SNHG16 can significantly enhance the proliferation of osteosarcoma cells. In addition, SNHG16, miR-205, and ZEB1 interact in a common pathway during the development and occurrence of osteosarcoma, providing novel targets for intervention in the treatment of osteosarcoma.
\end{abstract}




\section{Introduction}

Osteosarcoma is the most common primary malignant bone tumor of the skeletal system. It often occurs in patients aged 15-25 years, accounting for approximately $56 \%$ of bone tumors, making it the third most common malignant tumor in adolescents [1]. Osteosarcoma is characterized by high incidence, malignancy, and metastasis rates; therefore, its prognosis is poor. With the wide application of neoadjuvant chemotherapy and advancement of modern surgical techniques, the treatment of osteosarcoma has greatly improved [1], as shown by the increase in 5-year survival rate from $50 \%$ to $70 \%$ in the last 20 years [2]. However, multidrug resistance severely limits the development and use of neoadjuvant chemotherapy. Although progress has been made in molecular studies on osteosarcoma, much remains unclear about the molecular mechanisms involved in this disease.

With the development of DNA microarray and RNA sequencing techniques, it has been found that only $2 \%$ of transcripts in the genome encode proteins, whereas tens of thousands of transcripts have no encoding ability and are therefore considered genomic noise. However, accumulating evidence has suggested that these non-coding RNAs actually play important regulatory roles in gene expression [3-5]; non-coding RNAs longer than 200 base pairs in length are known as long non-coding RNAs (lncRNAs) [6]. In recent years, lncRNAs have been a research hotspot $[3,7]$, as they have been shown to play important roles in the development of tumors [6, 8-12].

Studies have also shown that IncRNAs are involved in various physiological processes such as the development, occurrence, invasion, and metastasis of a variety of tumors. There are many regulatory mechanisms used by lncRNAs, among which the competitive endogenous RNA (ceRNA) theory has been widely studied and recognized [13]. According to this theory, IncRNAs, mRNAs, pseudogenes, and other transcripts can competitively bind to microRNA (miRNA) through miRNA response elements (MREs), thereby reducing the number of miRNAs that bind to mRNAs and regulating the expression of downstream target genes of miRNAs at the posttranscriptional level. The important biological functions of lncRNAs have been gradually recognized $[3,10,14]$, but their roles in osteosarcoma have not yet been clarified.

In this study, differentially expressed IncRNAs in osteosarcoma and paracarcinoma tissues were identified by screening an IncRNA microarray, and candidate lncRNAs were verified by quantitative real-time PCR (qRT-PCR). The lncRNA small nucleolar RNA host gene 16 (SNHG16), which had the largest fold change, was selected as the focus of this study. A series of bioinformatics and molecular biological methods were adopted to investigate the interaction among lncRNA SNHG16, miRNA, and miRNA target genes during the development and occurrence of osteosarcoma, and to clarify the underlying molecular mechanisms. The results of this study may provide novel targets for intervention in the treatment of osteosarcoma and a theoretical basis for optimizing treatment strategies.

\section{Materials and Methods}

Tissue specimens

In this study, 20 osteosarcoma and paracarcinoma tissue samples were selected from osteosarcoma patients who received surgery at the Third Affiliated Hospital of Suzhou University between 2009 and 2014, and were stored in liquid nitrogen at $-80^{\circ} \mathrm{C}$. The collection of clinical specimens was approved by the Ethics Committee of the Third Affiliated Hospital of Suzhou University according to the Declaration of Helsinki. All participants provided written informed consent. 


\section{Cellular Physiology Cell Physiol Biochem 2018;51:429-440

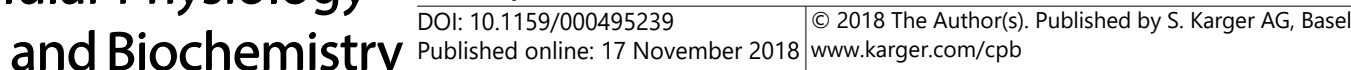 \\ Zhu et al.: Effects of LncRNA SNHG16 on Osteosarcoma}

\section{LncRNA detection by chip microarray}

The expression of IncRNAs in osteosarcoma and paracarcinoma tissues was analyzed using the Arraystar Human LncRNA Array v3.0 (Arraystar, Inc., Rockville, MD), which includes 33, 045 lncRNAs and 30,215 coding transcripts, according to the manufacturer's instructions. Statistical analyses were performed on the data. The differentially expressed IncRNAs in the two groups were selected based on a minimum 4-fold change in gene expression between osteosarcoma and paracarcinoma tissues, with $P$ values less than 0.01 according to $t$-tests.

\section{Cell culture and transfection}

The osteosarcoma cell lines (MG-63, U2OS, SAOS2, HOS, and OB3) used in this study were purchased from the American Type Culture Collection (Manassas, VA). SNHG16-overexpressing plasmid, SNHG16 small interfering RNAs (siRNAs), and negative controls were purchased from Sigma-Aldrich (St. Louis, MO). MiR205 mimics, miR-205 inhibitors, and negative controls were purchased from RiboBio (Guangzhou, China). Cells were cultured in RPMI- 1640 medium containing $10 \%$ fetal bovine serum in a $5 \% \mathrm{CO}_{2}$ atmosphere at $37^{\circ} \mathrm{C}$, and were transfected with Lipofectamine 2000 (Thermo Fisher Scientific, Waltham, MA) according to the manufacturer's instructions. The transfection efficiency was observed under a fluorescence microscope $24 \mathrm{~h}$ after transfection. Total RNA from tissues and cells was extracted using TRIzol reagent (Invitrogen, Carlsbad, CA). cDNA was synthesized from total RNA using the PrimerScript RT Reagent Kit (TaKaRa Bio, Tokyo, Japan). MiRNAs from total RNA were reverse transcribed using the Prime-Script miRNA cDNA Synthesis Kit (TaKaRa Bio) and subjected to subsequent amplification using the SYBR Green dye method on the 7900HT FAST Real-Time PCR System (Applied Biosystems, Foster City, CA). GAPDH was used as the internal reference for mRNA and U6 was the internal reference for miRNAs. The mRNA and miRNA expression levels were calculated using the $2^{-\Delta \Delta c t}$ method.

Prediction and validation of the binding of SNHG16 to miRNA-205, and prognosis and validation of miRNA-205 target genes

Three databases, namely, miRcode (http://www.mircode.org/), starBase (http://starbase.sysu. edu.cn/index.php), and RegRNA (http://regrna2.mbc.nctu.edu.tw/index.html), were used to predict the miRNAs that could bind to IncRNAs using the principle of base complementary pairing. The binding ability of IncRNAs to candidate miRNAs was verified by the luciferase reporter assay. The wild-type and mutanttype luciferase reporter plasmids psiCheck2-SNHG16-Luc and psiCheck2-SNHG16-MUT-Luc, respectively, were constructed. U2OS cells were co-transfected with miR-205 mimic, psiCheck2-SNHG16-Luc, and psiCheck2-SNHG16-MUT-Luc vectors. Finally, the luciferase activities in the experimental and control groups were detected using a luciferase reporter gene assay kit. Three databases, namely, TargetScan (http:// www.targetscan.org/vert_71/), miRDB (http://www.mirdb.org/), and RNA22 (https://cm.jefferson.edu/ rna22/), were used to predict the miR-205 target genes. The wild-type and mutant-type luciferase reporter plasmids psiCheck2-ZEB1-Luc and psiCheck2-ZEB1-MUT-Luc, respectively, were constructed. U2OS cells were co-transfected with miR-205 mimic, psiCheck2-ZEB1-Luc, and psiCheck2-ZEB1-MUT-Luc vectors, and the luciferase activities in the experimental and control groups were detected using a luciferase reporter gene assay kit.

\section{Cell Counting Kit-8 assay}

Cell proliferation was detected with the Cell Counting Kit-8 (CCK-8) assay. After transfection for $24 \mathrm{~h}$, cells from each group were collected and seeded into a 96-well plate. Three triplicate wells were used for $0,24,48,72$, and $96 \mathrm{~h}$ in each group. After cell adherence, $10 \mu \mathrm{L}$ CCK- 8 solution was added to each well and cells were cultured at $37^{\circ} \mathrm{C}$ for $4 \mathrm{~h}$. Cell viability was measured with a microplate absorbance reader (BioRad Laboratories, Hercules, CA) at $450 \mathrm{~nm}$.

\section{Statistical methods}

The Student's $t$-test was used for data comparison between two groups, and analysis of variance and chi-square tests were used for data analysis among groups and for intergroup comparison, respectively. The correlation among variables was analyzed by Spearman's correlation analysis. SPSS 20.0 and GraphPad Prism 5 software were used for statistical analysis and creating graphs, respectively. P values less than 0.05 were considered statistically significant. 


\section{Cellular Physiology Cell Physiol Biochem 2018;51:429-440

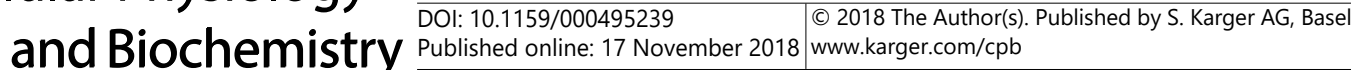 \\ Zhu et al.: Effects of LncRNA SNHG16 on Osteosarcoma}

\section{Results}

Screening of differentially expressed IncRNAs in osteosarcoma

LncRNA chip analysis was conducted for 5 pairs of osteosarcoma and paracarcinoma tissues, and differentially expressed lncRNAs were screened. Based on a minimal 4-fold change in IncRNA gene expression between osteosarcoma and paracarcinoma tissues with $P$ values less than 0.01 according to $t$-tests, we found that $65 \operatorname{lncRNAs}$ were upregulated and 13 were downregulated (Fig. 1A). In osteosarcoma, the 5 most significantly upregulated lncRNAs (ENSG00000250334, ENSG00000230234, ENSG00000163597, ENSG00000234147, and ENSG00000206195) and 5 most significantly downregulated lncRNAs (ENSG00000236990, ENSG00000247317, ENSG00000235837, ENSG00000224048, and ENSG00000266278) were selected for further verification. In 20 pairs of osteosarcoma and paracarcinoma tissues, we analyzed the expression of these 10 lncRNAs by qRT-PCR and found that ENSG00000163597 (IncRNA SNHG16) was the most highly expressed in osteosarcoma (Fig. 1B). Therefore, SNHG16 was selected for subsequent experiments. We analyzed the relationship between SNHG16 expression and tumor stage in 6 patients with stage I, 8 patients with stage II, and 6 patients with stage III disease. SNHG16 expression in stage I was significantly lower than expression in stages II and III (Fig. 1C). Next, we evaluated the difference in SNHG16 expression between normal osteoblasts (OB3) and osteosarcoma cell lines (MG-63, U2OS, SAOS2, and HOS), and found that SNHG16 expression was significantly higher in osteosarcoma cells than in normal osteoblasts ( $<<0.01$, Fig. 1D).

SNHG16 regulates the proliferation of osteosarcoma cells

We analyzed the expression levels of SNHG16 in MG-63, U2OS, SAOS2, and HOS osteosarcoma cell lines and found that they were higher in MG-63 and HOS cells than in U2OS and SAOS2 cells (Fig. 1C). Transfection of SNHG16 siRNAs si1 and si2 in MG-63 and HOS cell lines led to the downregulation of SNHG16 expression, whereas transfection of the pcDNA3.1-SNHG16 overexpressing vector into U2OS and SAOS2 cell lines led to SNHG16 upregulation. Knockdown and overexpression efficiencies were verified by qRT-PCR (P < 0.001, Fig. 2A, B, respectively). After SNHG16 downregulation in MG-63 and HOS cells, the cell proliferation was significantly decreased ( $<<0.001$, Fig. 2C, D). After SNHG16 overexpression in U2OS and SAOS2 cells, the cell proliferation was significantly enhanced $(\mathrm{P}<0.001$, Fig. $2 \mathrm{E}$, F). Because osteosarcoma cell lines have low apoptotic rates, we selected only MG-63 and HOS for apoptosis analysis. Apoptosis was significantly increased in MG-63 and HOS cells in response to the downregulation of SNHG16 expression compared to the controls (Fig. $2 \mathrm{G}, \mathrm{H}$ ). In addition, the downregulation of SNHG16 in MG-63 and HOS cells increased the expression of apoptosis-related proteins including cleaved caspase- 3 and cleaved poly (ADPribose) polymerase (Fig. 2I-K). Thus, we concluded that SNHG16 had significant regulatory effects on the proliferation of osteosarcoma cells.

\section{Screening and validation of miRNAs regulated by SNHG16}

Three bioinformatics databases (miRcode, starBase, and RegRNA) were jointly used to predict miRNAs that could bind to SNHG16 using the base complementary pairing principle (Fig. 3A). A total of 5 miRNAs (let-7, miR-200a, miR-141, miR-205, and miR-132) discovered in all three databases were found to bind SNHG16. Thus, the expression of these 5 miRNAs was subsequently analyzed by qRT-PCR in 20 osteosarcoma tissue samples. Only miR-205 expression was negatively correlated with SNHG16 expression $(r=-0.68, \mathrm{P}<0.001$, Fig. 3B), suggesting that miR-205 may be regulated by SNHG16. To further verify that SNHG16 was a target of miR-205, wild-type and mutant-type SNHG16 sequences were cloned into the luciferase reporter plasmid psiCheck2 and then co-transfected with miR-205 mimic into U2OS cells for the luciferase reporter gene assay (Fig. 3C). The overexpression of miR205 significantly reduced the luciferase activity of wild-type SNHG16 $(\mathrm{P}<0.001)$ without decreasing the luciferase activity of mutant-type SNHG16 or empty vector (Fig. 3D), further 


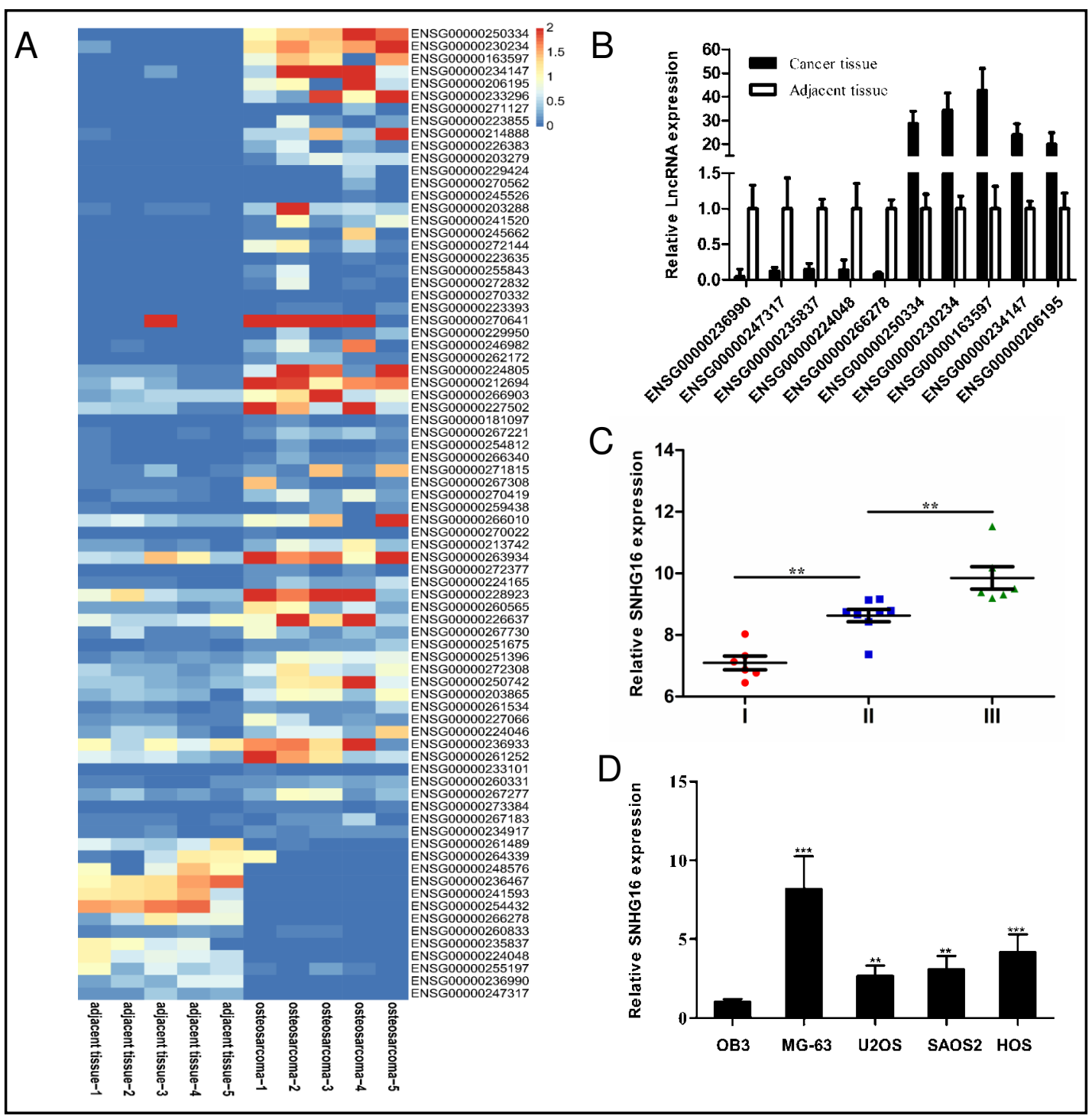

Fig. 1. Screening of differentially expressed lncRNAs in osteosarcoma. (A) LncRNA chip analysis. Compared with para-carcinoma tissues, 65 IncRNAs are up-regulated in osteosarcoma tissues, and 13 lncRNAs are down-regulated (Fold change $>4.0$, t-test P<0.01). (B) qRT-PCR on 10 selected lncRNAs with the highest differential expression in osteosarcoma and para-carcinoma tissues. ENSG00000163597 (lncRNA SNHG16) is the most significantly up-regulated in osteosarcoma. (C) Relative SNHG16 expression in different cancer stage. (D) Detection of differences in lncRNA SNHG16 expression in normal osteoblasts (OB3) and osteosarcoma cell lines (MG-63, U20S, SAOS2, HOS) $\left({ }^{* *} \mathrm{P}<0.001,{ }^{* *} \mathrm{P}<0.01\right)$.

confirming that SNHG16 could bind directly to miR-205 and that the mutation site in the mutant-type vector was the binding site of miR-205. In addition, we detected the efficiency of miR-205 overexpression and knockdown (Fig. 3E, F).

\section{Screening and validation of miR-205 target genes}

To search for potential target genes of miR-205, three databases (TargetScan, miRDB, and RNA22) were used, and the most likely target genes were identified (claudin 11, cyclindependent kinase 14, acetyl-coenzyme A synthetase 1, cyclin J, and zinc finger E-box-binding homeobox 1 [ZEB1]). The expression of miR-205 was upregulated in MG-63 cells, while the expression of ZEB1 was decreased ( $<<0.001$, Fig. 4A). The expression of miR-205 was downregulated in U20S cells, while the expression of ZEB1 was increased $(\mathrm{P}<0.001$, Fig. 

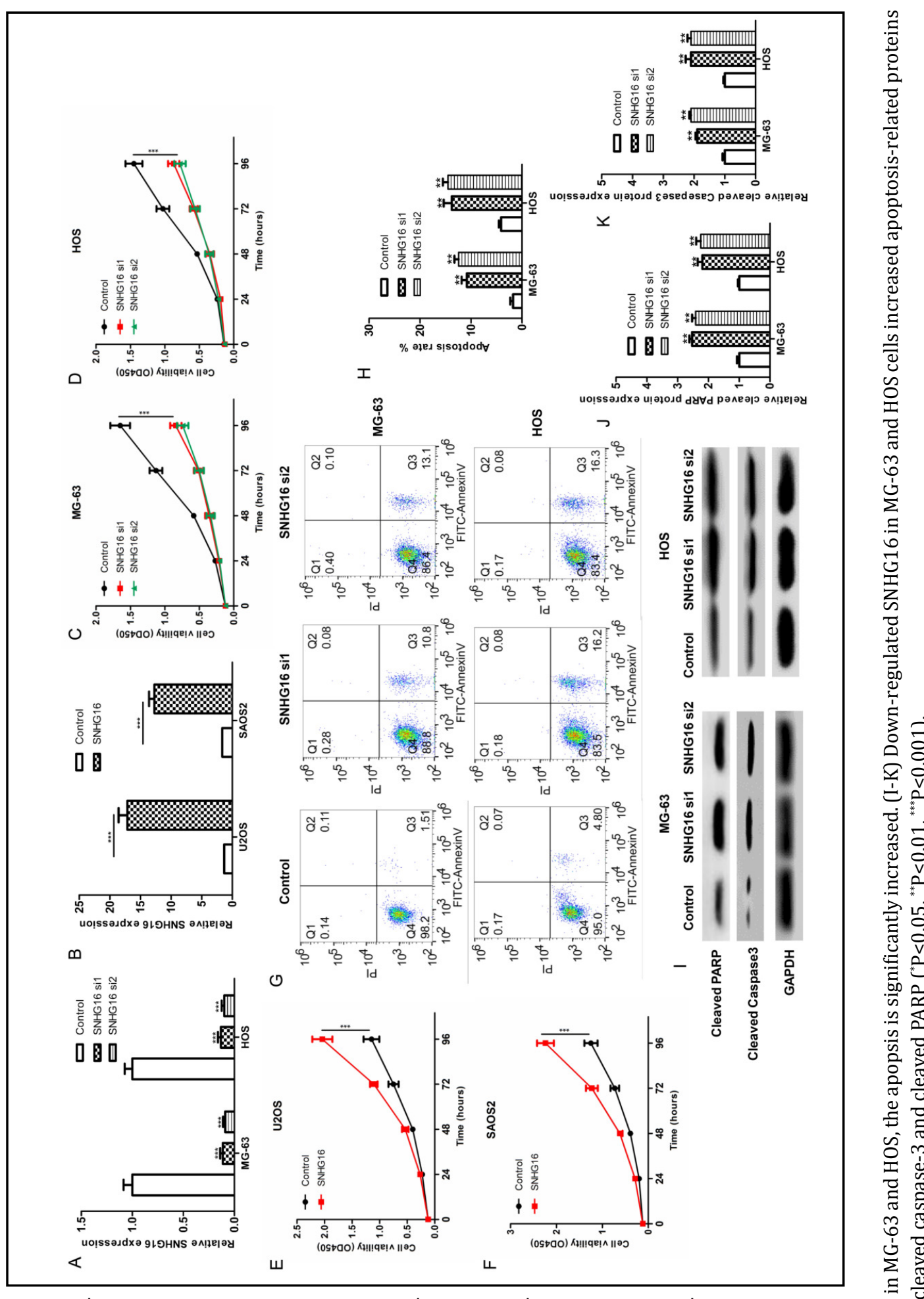

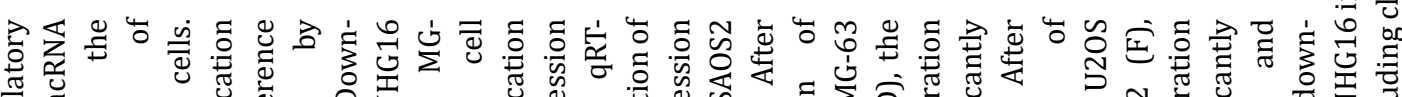

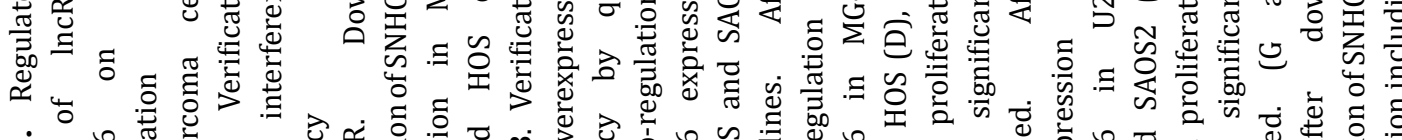

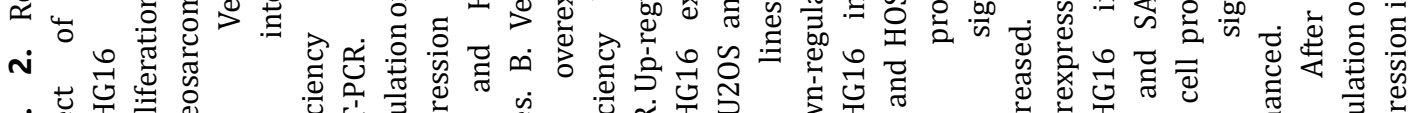

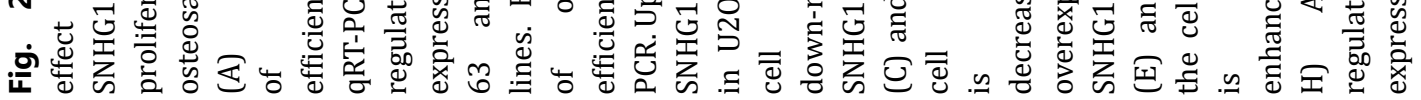
KARGER 


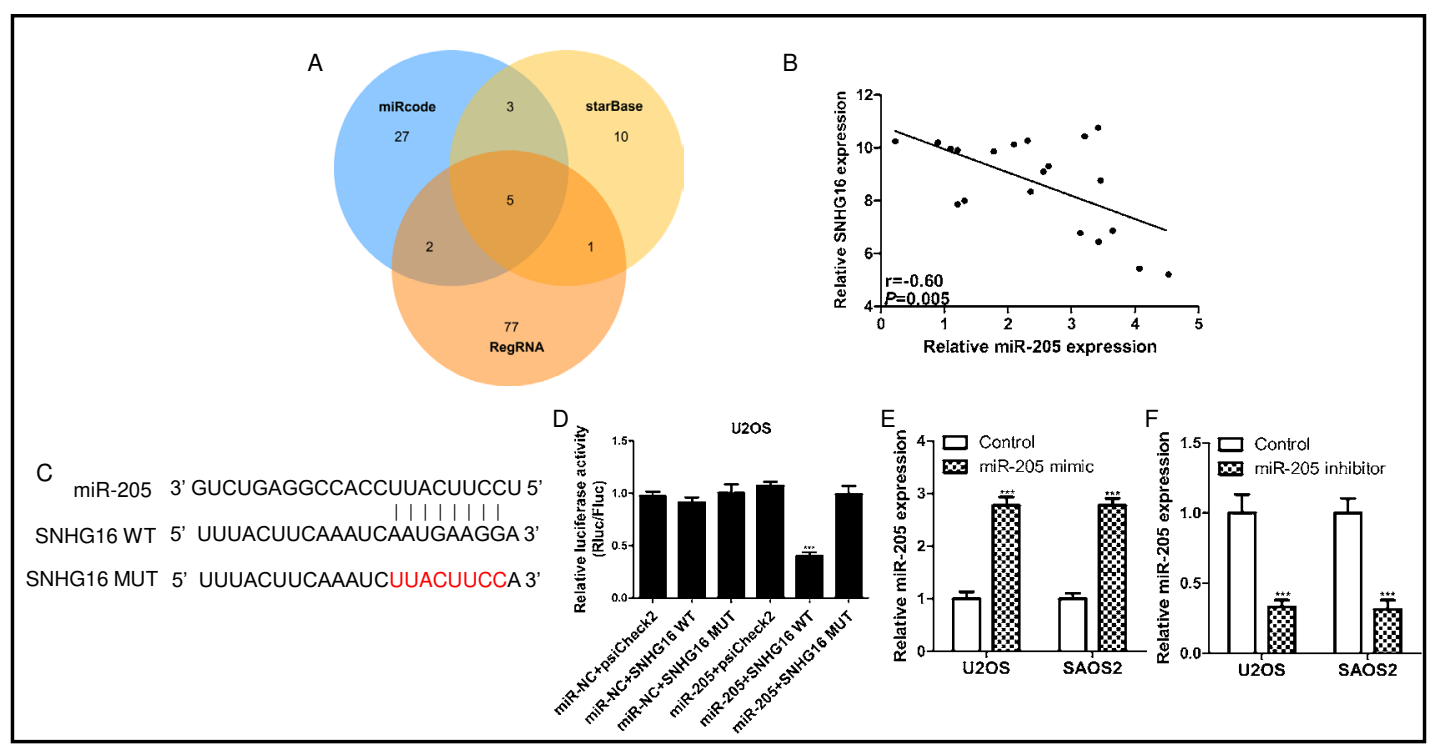

Fig. 3. Screening and validation of miRNAs regulated by SNHG16. (A) MiRcode, starBase and RegRNA are used to predict miRNAs that can bind to SNHG16. A total of 5 miRNAs, common to all 3 databases, can bind to SNHG16. (B) qRT-PCR shows that miR-205 expression is negatively correlated with SNHG16 expression in osteosarcoma tissues $(\mathrm{n}=20)(\mathrm{r}=-0.60, \mathrm{P}=0.005)$. (C) miR-205 and SNHG16 binding sequences and SNHG16 mutant sequences. (D) Luciferase reporter assay is used to verify the targeting effect of miR-205 on SNHG16 sequence. (E) Verification of interference efficiency by qRT-PCR. Up-regulation of miR-205 expression in U2OS and SAOS2 cell lines. (F) Verification of interference efficiency by qRT-PCR. Down-regulation of miR205 expression in U2OS and SAOS2 cell lines. $\left({ }^{* * *} \mathrm{P}<0.001\right)$.

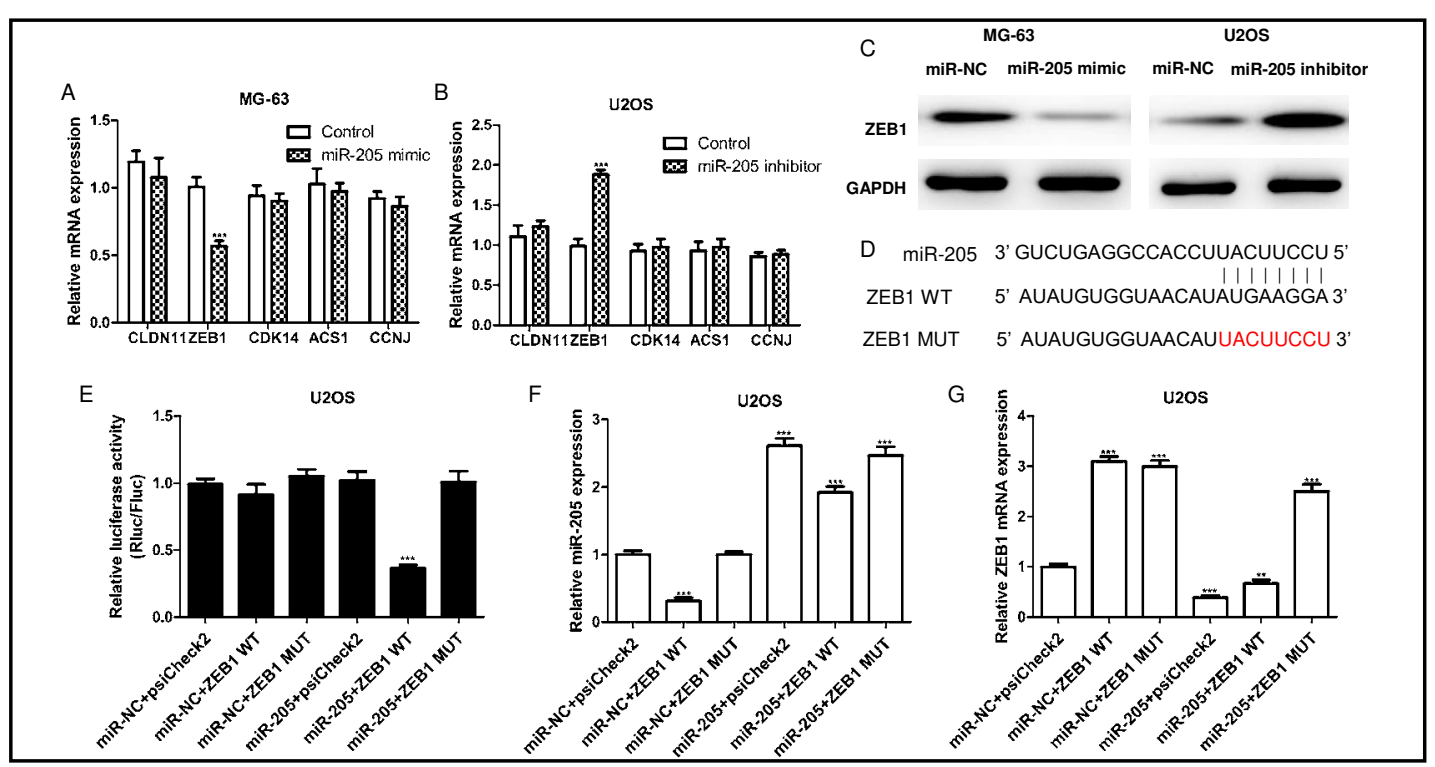

Fig. 4. Screening and validation of miR-205 target genes. (A) After the up-regulation of miR-205 expression in MG-63 cell line, the mRNA expression level of each candidate target genes is analysed by qRT-PCR $\left({ }^{* * *} \mathrm{P}<0.001\right)$. (B) After the down-regulation of miR-205 expression in U2OS cell line, the mRNA expression level of each candidate target genes is analysed by qRT-PCR $\left({ }^{* * *} \mathrm{P}<0.001\right)$. (C) ZEB1 protein expression after miR-205 overexpression or deactivation were detected by Western blotting. (D) miR-205 and ZEB1 3'UTR binding sequences and ZEB1 mutant sequences. (E) Luciferase reporter assay is used to verify the direct binding between miR-205 and ZEB1 $\left.{ }^{* * *} \mathrm{P}<0.001\right)$. (F) miR-205 expression in mRNA report is analysed by qRT-PCR $\left({ }^{* * *} \mathrm{P}<0.001\right)$. (G) ZEB1 mRNA expression in mRNA report is analysed by $\mathrm{qRT}$-PCR $\left({ }^{* *} \mathrm{P}<0.01\right.$, $\left.{ }^{* * *} \mathrm{P}<0.001\right)$. 


\section{Cellular Physiology Cell Physiol Biochem 2018;51:429-440 \begin{tabular}{l|l|l} 
and Biochemistry Published online: 17 November 2018 & $\begin{array}{l}\text { (c) } 2018 \text { The Author(s). Published by S. Karger AG, Basel } \\
\text { www.karger.com/cpb }\end{array}$ \\
\hline
\end{tabular} \\ Zhu et al.: Effects of LncRNA SNHG16 on Osteosarcoma}

4B). There were no significant changes in the expression of any other miR-205 candidate target genes, suggesting that ZEB1 was the target gene of miR-205. The same results were observed with ZEB1 protein (Fig. 4C). To perform luciferase reporter assays, the wild-type and mutant 3' untranslated region (3'UTR) sequences of ZEB1 were cloned into the luciferase reporter plasmid psiCheck2 and co-transfected with miR-205 mimic into U2OS cells (Fig. 4D). Transfection with wild-type ZEB1 luciferase reporter plasmid significantly reduced the luminescence intensity compared with the mutant-type ZEB1 luciferase reporter plasmid (P $<0.001$, Fig. 4E), indicating that miR-205 could bind directly to ZEB1, and that the miR-205 binding site in the ZEB1 3'UTR was the mutation site. We also evaluated the level of miR-205 after expression of reporter mRNA (Fig. 4F).

\section{SNHG16 regulates ZEB1 expression through competitive binding with miR-205}

To further investigate the regulatory effect of SNHG16 on ZEB1 expression, SNHG16 expression was knocked down in MG-63 and HOS cell lines by transfection with SNHG16 siRNA, which led to a significant increase in miR-205 expression $(\mathrm{P}<0.001$, Fig. 5A), while ZEB1 mRNA and protein expression was significantly decreased ( $<<0.001$, Fig. 5B, C). In parallel, SNHG16 expression was upregulated in U2OS and SAOS2 cell lines, which led to a significant decrease in miR-205 expression ( $\mathrm{P}<0.001$, Fig. 5D), while ZEB1 mRNA and protein expression was significantly increased ( $P<0.001$, Fig. 5E, F). Moreover, in 20 pairs of osteosarcoma tissues, there was a negative correlation between SNHG16 and miR-205 expression $(r=-0.60, \mathrm{P}=0.005$, Fig. 3B), a negative correlation between miR-205 and ZEB1 expression ( $r=-0.54, \mathrm{P}=0.014$, Fig. $5 \mathrm{G})$, and a positive correlation between SNHG16 and ZEB1 expression $(r=0.80, \mathrm{P}<0.001$, Fig. 5H). These data showed that SNHG16, miR-205, and ZEB1 formed a ceRNA, and that SNHG16 regulated ZEB1 expression by competitively binding miR-205. In addition, the regulation of osteosarcoma cell proliferation by SNHG16 was miR-205-dependent.

To determine whether the regulatory role of SNHG16 on the proliferation of osteosarcoma cells was dependent on miR-205, a cell proliferation rescue assay was performed. MG-63 and HOS cell lines with high SNHG16 expression were transfected with control siRNA+miRNC, SNHG16 si1+miR-NC, control siRNA+miR-205 inhibitor, or SNHG16 si1+miR-205 inhibitor. The results demonstrated that downregulation of miR-205 expression promoted cell proliferation in both cell lines, whereas downregulation of SNHG16 expression suppressed cell proliferation ( $\mathrm{P}<0.001$, Fig. 6A, B). Downregulation of both SNHG16 and miR-205 expression had no significant effect on the proliferation capacity of osteosarcoma cells with only knockdown of miR-205 (P > 0.05, Fig. 6A, B). U2OS and SAOS2 cell lines with relatively low SNHG16 expression were transfected with control plasmid+miR-NC, SNHG16 overexpressing plasmid+miR-NC, control plasmid+miR-205 mimic, and SNHG16 overexpressing plasmid+miR-205 mimic. The upregulation of both SNHG16 and miR-205 expression had no significant effect on the proliferation capacity of osteosarcoma cells with only overexpression miR-205 (P > 0.05, Fig. 6C, D). Therefore, we confirmed that SNHG16 promoted cell proliferation through miR-205. To investigate whether the regulatory role of miR-205 on the proliferation of osteosarcoma cells was dependent on ZEB1, a cell proliferation rescue assay was performed. MG-63 and HOS cell lines with high ZEB1 expression were transfected with control siRNA+miR-NC, ZEB1 si1+miR-NC, control siRNA+miR-205 inhibitor, or ZEB1 si1+miR-205 inhibitor. The downregulation of both ZEB1 and miR-205 expression had no significant effect on the proliferation capacity of osteosarcoma cells with only ZEB1 knockdown (Fig. 7A, B). U2OS and SAOS2 cell lines with relatively low expression of ZEB1 were transfected with control plasmid+miR-NC, ZEB1 overexpressing plasmid+miR$\mathrm{NC}$, control plasmid+miR-205 mimic, and ZEB1 overexpressing plasmid+miR-205 mimic. The upregulation of both ZEB1 and miR-205 expression had no significant effect on the proliferation capacity of osteosarcoma cells with only ZEB1 overexpression (Fig. 7C, D). These data confirmed that miR-205 promoted cell proliferation in ZEB1-dependent manner. 


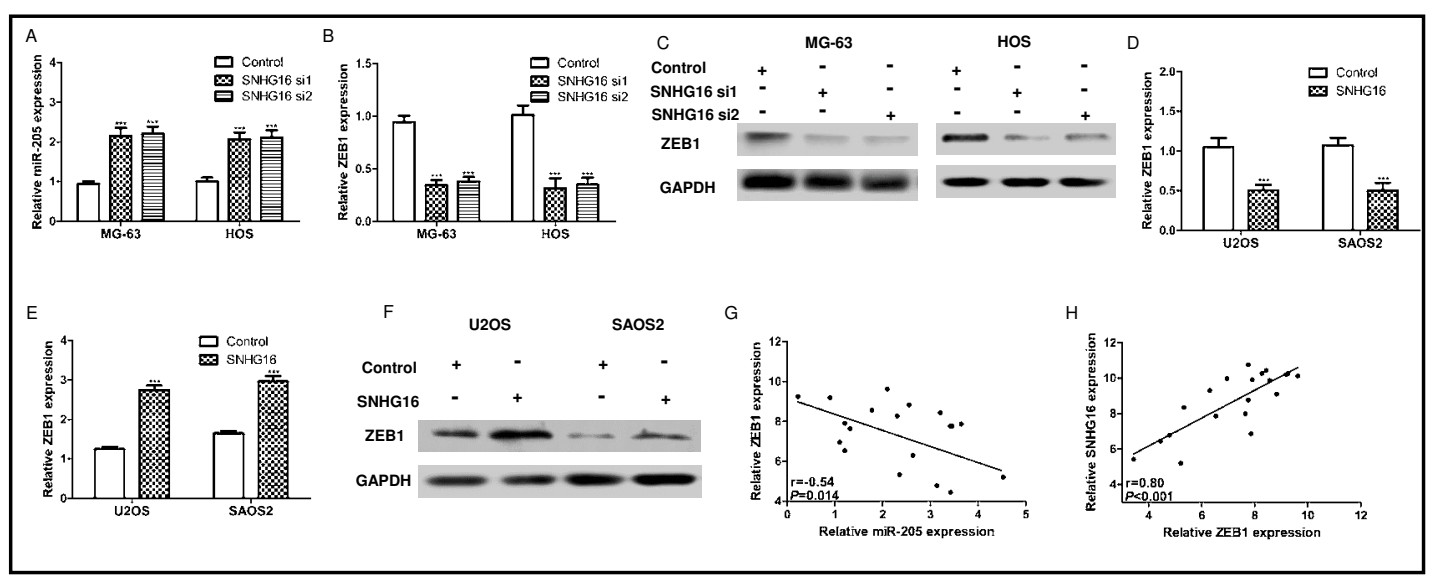

Fig. 5. SNHG16 regulates miR-205 and ZEB1 expression in osteosarcoma cells. After the down-regulation of SNHG16 expression in MG-63 and HOS cell lines, (A) miR-205 expression is significantly increased $\left({ }^{* * *} \mathrm{P}<0.001\right)$, while $(\mathrm{B}-\mathrm{C})$ ZEB1 mRNA and protein expression is significantly decreased $\left({ }^{* * *} \mathrm{P}<0.001\right)$. After the up-regulation of SNHG16 expression in U2OS and SAOS2 cell lines, (D) miR-205 expression is significantly decreased $\left({ }^{* * *} \mathrm{P}<0.001\right)$, while (E-F) ZEB1 mRNA and protein expression is significantly increased $\left({ }^{* *} \mathrm{P}<0.001\right)$. (G) There is a negative correlation between miR-205 and ZEB1 expression levels in osteosarcoma tissues $(\mathrm{n}=20)(\mathrm{r}=-0.54, \mathrm{P}=0.014)$. $(\mathrm{H})$ There is a positive correlation between SNHG16 and ZEB1 expression levels in osteosarcoma tissues $(n=20)(r=0.80, P<0.001)$.

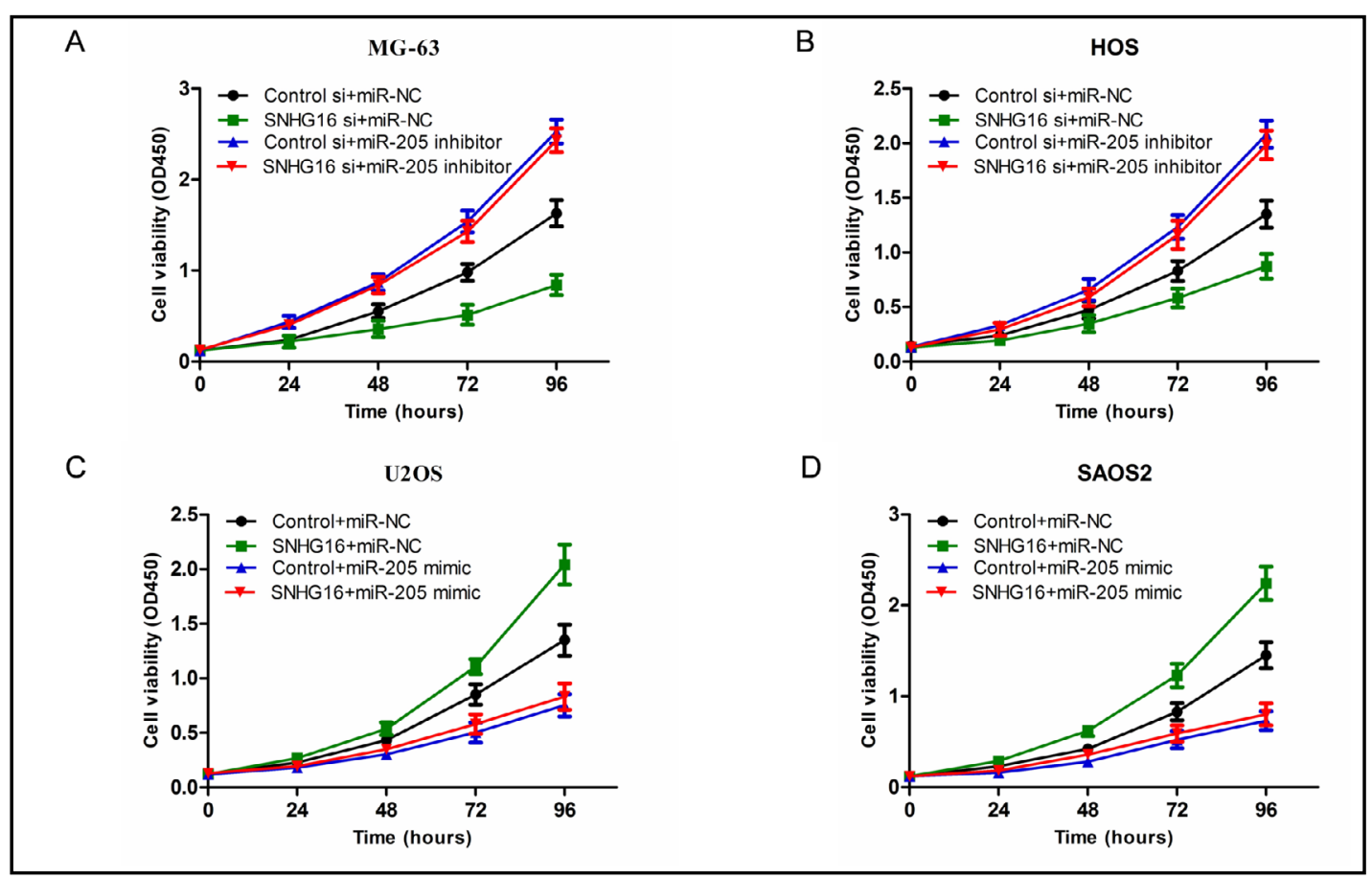

Fig. 6. The regulatory effect of SNHG16 on the proliferation of osteosarcoma cells is miR-205-dependent. (A) MG-63 and (B) HOS cells are transfected with Control siRNA+miR-NC, SNHG16 si1+miR-NC, Control siRNA+miR-205 inhibitor or SNHG16 si1+miR-205 inhibitor, and the cell proliferation in each group is detected by CCK-8. (C) U2OS and (D) SAOS2 cells are transfected with Control plasmid+miR-NC, SNHG16 overexpressing plasmid+miR-NC, Control plasmid+miR-205 mimic and SNHG16 overexpressing plasmid+miR-205 mimic, and the cell proliferation in each group is detected by CCK-8. 


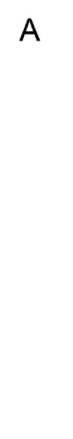

C
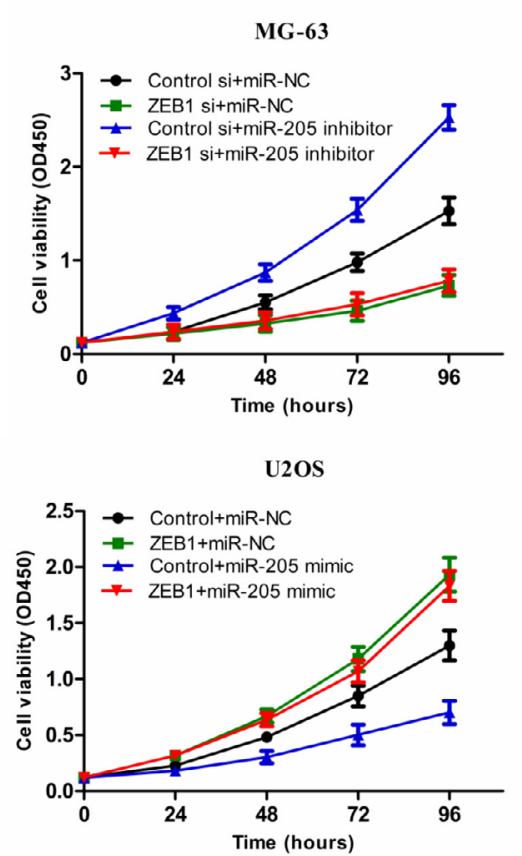

B

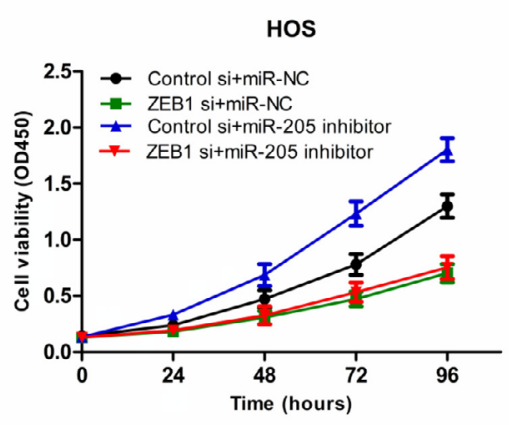

D

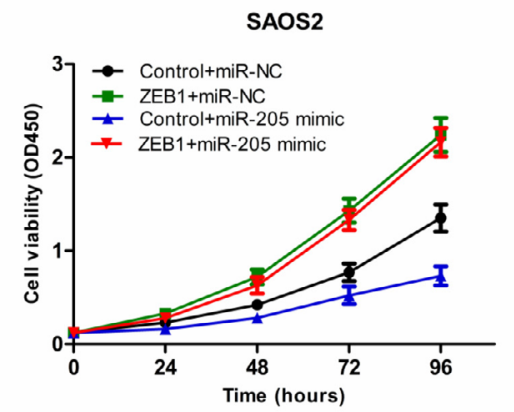

Fig. 7. The regulatory effect of miR-205 on the proliferation of osteosarcoma cells is ZEB1-dependent. (A) MG-63 and (B) HOS cells are transfected with Control siRNA+miR-NC, ZEB1 si1+miR-NC, Control siRNA+miR-205 inhibitor or ZEB1 si1+miR-205 inhibitor, and the cell proliferation in each group is detected by CCK-8. (C) U2OS and (D) SAOS2 cells are transfected with Control +miR-NC, ZEB1 overexpressing plasmid+miR-NC, Control+miR-205 mimic and ZEB1 overexpressing plasmid+miR-205 mimic, and the cell proliferation in each group is detected by CCK-8.

\section{Discussion}

In the human genome, $90 \%$ of DNA sequences do not encode proteins. Research studies have shown that these non-protein-coding sequences play important roles in gene regulation at multiple levels, among which short non-coding RNAs with transcripts of 18-25 nucleotides in length include miRNAs, siRNAs, and Piwi-interaction RNAs. The biological function and mechanism of miRNAs have been studied widely. LncRNAs, a type of non-coding RNA lacking biological functions, have garnered increasing attention in recent years. Studies have shown that they have various biological functions in gene transcription and translation, cell differentiation and development, inheritance, and epigenetic inheritance $[3,7]$, and especially in the development and occurrence of tumors, where they play key regulatory functions $[6,8-12,15]$. LncRNAs are abnormally expressed in many tumors. In particular, there has been an increasing number of studies on lncRNAs in the field of osteosarcoma. Some lncRNAs are tumor-promoting genes. For example, Yan et al. [15] found that the lncRNA colon cancer associated transcript 2 significantly promotes the proliferation and migration of osteosarcoma cells by increasing the expression of $\mathrm{N}$-cadherin, vimentin, and snail. Ye et al. [16] found that the prognosis of patients with high expression of the lncRNA NNT-AS1 in osteosarcoma is poor, and that the upregulation of nicotinamide nucleotide transhydrogenase-antisense RNA 1 can increase the proliferation of osteosarcoma cells. On the other hand, some lncRNAs are cancer suppressor genes. For example, Han et al. [17] found in a study on osteosarcoma that lncRNA-p21 can inhibit the expression of miR-130b, thereby inhibiting the proliferation of tumor cells. Wang et al. [18] found that silencing the expression of IncRNA growth-arrest specific 5 in osteosarcoma cell lines upregulates the expression of miR-203a, thus promoting the proliferation and metastasis of osteosarcoma cells. Therefore, IncRNAs play important roles in the development and occurrence of osteosarcoma; however, their specific mechanisms of action remain to be further clarified.

ceRNA regulatory mechanisms are complex regulatory relationships among different RNAs, including mRNAs encoding proteins and pseudogenes, lncRNAs, and circular RNAs 


\section{Cellular Physiology Cell Physiol Biochem 2018;51:429-440 \begin{tabular}{l|l|l} 
and Biochemistry Published online: 17 November 2018 & $\begin{array}{l}\text { (c) } 2018 \text { The Author(s). Published by S. Karger AG, Basel } \\
\text { www.karger.com/cpb }\end{array}$ \\
\hline
\end{tabular} \\ Zhu et al.: Effects of LncRNA SNHG16 on Osteosarcoma}

without protein coding functions, and they contain common miRNA-binding sites that can jointly exert cancer-promoting or tumor-suppressing effects by competitively binding to MREs [16, 17], thereby alleviating the inhibitory effects of miRNAs on their target genes and inhibiting or promoting tumor progression [18]. The ceRNA theory provides a new perspective to further explore the molecular mechanism of SNHG16 in the development and occurrence of osteosarcoma. By using chip microarray screening combined with the validation of differentially expressed candidate lncRNAs, the IncRNA SNHG16 had the largest fold change. SNHG16 was highly expressed in osteosarcoma tissues and cell lines. Previous studies have shown that SNHG16 is upregulated and acts as a potential oncogene in malignant tumors [19-21]. Bioinformatics predicted that there are many miRNA-binding sites in the SNHG16 sequence, and the luciferase reporter assay confirmed the direct interaction between SNHG16 and miR-205. This study also showed that there was a significant negative correlation between the expression ofSNHG16 and miR-205 in osteosarcoma tissues. Previous studies have demonstrated that miR-205 acts as a tumor suppressor in osteosarcoma [2224]. Subsequently, we successfully predicted and validated ZEB1 as a miR-205 target gene. ZEB1 plays an important regulatory role in the progression of cell cycle, cell growth, and differentiation, and its deletion promotes cancer cell proliferation. Shen et al. [28] found that ZEB1 is highly expressed in osteosarcoma and that the high expression of ZEB1 can promote the proliferation and metastasis of osteosarcoma cells. We also found that SNHG16 has a regulatory effect on ZEB1, as when SNHG16 was upregulated in osteosarcoma, miR205 expression was decreased and ZEB1 expression was increased. After downregulation of SNHG16 expression, miR-205 expression was significantly increased and ZEB1 expression was significantly decreased. In addition, the expression of SNHG16, miR-205, and ZEB1 was significantly correlated in 20 samples of osteosarcoma tissues. Taken together, our data showed that SNHG16, miR-205, and ZEB1 formed a ceRNA, and that SNHG16 regulated ZEB1 expression by competitively binding to miR-205. Rescue assays confirmed that the effects of SNHG16 on the proliferation of osteosarcoma cells were dependent on miR-205.

\section{Conclusion}

This study demonstrated that SNHG16 can significantly enhance the proliferation of osteosarcoma cells and that SNHG16, miR-205, and ZEB1 interact in a common pathway during the development and occurrence of osteosarcoma. The results of this study may provide novel targets for intervention in the treatment of osteosarcoma and a theoretical basis for the optimization of therapeutic strategies.

\section{Disclosure Statement}

The authors declare no conflicts of interest.

\section{References}

1 Mirabello L, Troisi RJ, Savage SA: Osteosarcoma incidence and survival rates from 1973 to 2004: data from the Surveillance, Epidemiology, and End Results Program. Cancer 2009;115:1531-1543.

-2 Bielack SS, Kempf-Bielack B, Delling G, Exner GU, Flege S, Helmke K, Kotz R, Salzer-Kuntschik M, Werner M, Winkelmann W, Zoubek A, Jurgens H, Winkler K: Prognostic factors in high-grade osteosarcoma of the extremities or trunk: an analysis of 1, 702 patients treated on neoadjuvant cooperative osteosarcoma study group protocols. J Clin Oncol 2002;20:776-790.

Wang KC, Chang HY: Molecular mechanisms of long noncoding RNAs. Mol Cell 2011;43:904-914.

Wang X, Arai S, Song X, Reichart D, Du K, Pascual G, Tempst P, Rosenfeld MG, Glass CK, Kurokawa R: Induced ncRNAs allosterically modify RNA-binding proteins in cis to inhibit transcription. Nature 2008;454:126130. 


\section{Cellular Physiology Cell Physiol Biochem 2018;51:429-440 and Biochemistry \begin{tabular}{l|l} 
DOI: 10.1159/000495239 2018 The Author(s). Published by S. Karger AG, Basel \\
(c)
\end{tabular} \\ Zhu et al.: Effects of LncRNA SNHG16 on Osteosarcoma}

5 Shao Y, Zhang D, Li X, Yang J, Chen L, Ning Z, Xu Y, Deng G, Tao M, Zhu Y, Jiang J: MicroRNA-203 Increases Cell Radiosensitivity via Directly Targeting Bmi-1 in Hepatocellular Carcinoma. Mol Pharm 2018;15:32053215.

6 Prensner JR, Chinnaiyan AM: The emergence of lncRNAs in cancer biology. Cancer Discov 2011;1:391-407.

7 Mercer TR, Dinger ME, Mattick JS: Long non-coding RNAs: insights into functions. Nat Rev Genet 2009;10:155-159.

8 Du Z, Fei T, Verhaak RG, Su Z, Zhang Y, Brown M, Chen Y, Liu XS: Integrative genomic analyses reveal clinically relevant long noncoding RNAs in human cancer. Nat Struct Mol Biol 2013;20:908-913.

-9 Gupta RA, Shah N, Wang KC, Kim J, Horlings HM, Wong DJ, Tsai MC, Hung T, Argani P, Rinn JL, Wang Y, Brzoska P, Kong B, Li R, West RB, van de Vijver MJ, Sukumar S, Chang HY: Long non-coding RNA HOTAIR reprograms chromatin state to promote cancer metastasis. Nature 2010;464:1071-1076.

10 Gutschner T, Hammerle M, Eissmann M, Hsu J, Kim Y, Hung G, Revenko A, Arun G, Stentrup M, Gross M, Zornig M, MacLeod AR, Spector DL, Diederichs S: The noncoding RNA MALAT1 is a critical regulator of the metastasis phenotype of lung cancer cells. Cancer Res 2013;73:1180-1189.

11 Liu B, Sun L, Liu Q Gong C, Yao Y, Lv X, Lin L, Yao H, Su F, Li D, Zeng M, Song E: A cytoplasmic NF-kappaB interacting long noncoding RNA blocks IkappaB phosphorylation and suppresses breast cancer metastasis. Cancer Cell 2015;27:370-381.

12 Gutschner T, Diederichs S: The hallmarks of cancer: a long non-coding RNA point of view. RNA Biol 2012;9:703-719.

13 Salmena L, Poliseno L, Tay Y, Kats L, Pandolfi PP: A ceRNA hypothesis: the Rosetta Stone of a hidden RNA language? Cell 2011;146:353-358.

14 Yoon JH, Abdelmohsen K, Gorospe M: Functional interactions among microRNAs and long noncoding RNAs. Semin Cell Dev Biol 2014;34:9-14.

-15 Wang W, Li X, Meng FB, Wang ZX, Zhao RT, Yang CY: Effects of the Long Non-Coding RNA HOST2 On the Proliferation, Migration, Invasion and Apoptosis of Human Osteosarcoma Cells. Cell Physiol Biochem 2017;43:320-330.

16 Tay Y, Rinn J, Pandolfi PP: The multilayered complexity of ceRNA crosstalk and competition. Nature 2014;505:344-352.

17 Kartha RV, Subramanian S: Competing endogenous RNAs (ceRNAs): new entrants to the intricacies of gene regulation. Front Genet 2014;5:8.

18 Karreth FA, Pandolfi PP: ceRNA cross-talk in cancer: when ce-bling rivalries go awry. Cancer Discov 2013;3:1113-1121.

19 Lu YF, Cai XL, Li ZZ, Lv J, Xiang YA, Chen JJ, Chen WJ, Sun WY, Liu XM, Chen JB: LncRNA SNHG16 Functions as an Oncogene by Sponging MiR-4518 and Up-Regulating PRMT5 Expression in Glioma. Cell Physiol Biochem 2018;45:1975-1985.

20 Cai C, Huo Q Wang X, Chen B, Yang Q: SNHG16 contributes to breast cancer cell migration by competitively binding miR-98 with E2F5. Biochem Biophys Res Commun 2017;485:272-278.

-21 Zhu H, Zeng Y, Zhou CC, Ye W: SNHG16/miR-216-5p/ZEB1 signal pathway contributes to the tumorigenesis of cervical cancer cells. Arch Biochem Biophys 2018;637:1-8.

22 Zhang C, Long F, Wan J, Hu Y, He H: MicroRNA-205 acts as a tumor suppressor in osteosarcoma via targeting RUNX2. Oncol Rep 2016;35:3275-3284.

23 Yang G, Zhang P, Lv A, Liu Y, Wang G: MiR-205 functions as a tumor suppressor via targeting TGF-alpha in osteosarcoma. Exp Mol Pathol 2016;100:160-166.

-24 Wang L, Shan M, Liu Y, Yang F, Qi H, Zhou L, Qiu L, Li Y: miR-205 suppresses the proliferative and migratory capacity of human osteosarcoma Mg-63 cells by targeting VEGFA. Onco Targets Ther 2015;8:2635-2642.

-25 Zhang C, Long F, Wan J, Hu Y, He H: Microrna-205 acts as a tumor suppressor in osteosarcoma via targeting runx2. Oncol Rep 2016;35:3275-3284.

26 Yang G, Zhang P, Lv A, Liu Y, Wang G: Mir-205 functions as a tumor suppressor via targeting tgf-alpha in osteosarcoma. Exp Mol Pathol 2016;100:160-166.

27 Wang L, Shan M, Liu Y, Yang F, Qi H, Zhou L, Qiu L, Li Y: Mir-205 suppresses the proliferative and migratory capacity of human osteosarcoma mg-63 cells by targeting vegfa. Onco Targets Ther 2015;8:2635-2642.

28 Shen A, Zhang Y, Yang H, Xu R, Huang G: Overexpression of zeb1 relates to metastasis and invasion in osteosarcoma. J Surg Oncol 2012;105:830-834. 